\title{
Primer especímen de la \\ Colección de Mamíferos de la Universidad del Quindío (CMUQ): registro de Eptesicus chiriquinus (Chiroptera: Vespertillionidae) por atropellamiento en la Autopista del Café, Quindío, Colombia
}

\author{
First specimen of the \\ Mammal Collection of The Universidad del Quindío (CMUQ): \\ record of Eptesicus chiriquinus (Chiroptera: Vespertillionidae) hit by a \\ car at the Autopista del Café, Quindío, Colombia
}

\author{
Diego Fernando Gutiérrez-Cifuentes*, Anderson Brand-Alape*, Hugo Mantilla-Meluk* \\ Resumen
}

\begin{abstract}
Se documenta un macho juvenil del murciélago vespertilionido Eptesicus chiriquinus, recuperado el 15 de agosto de 2013 en la Autopista del Café, El Roble, Quindío, el cual fue aparentemente atropellado. El espécimen corresponde al primer ejemplar de la Colección Mastozoológica de la Universidad del Quindío (CMUQ) y fue identificado con número de catálogo CMUQ-001. A pesar de que la especie ya ha sido reportada para el departamento, este espécimen constituye el primer registro verificado mediante un procedimiento científico en una publicación revisada por pares. Finalmente, se discute sobre la importancia del inicio de la colección de mamíferos en esta institución.
\end{abstract}

Palabras clave: Colección científica, Eptesicus chiriquinus, Mamíferos Quindío, Vespertillionidae.

\begin{abstract}
We document a male juvenile specimen of the vespertillionid bat Eptesicus chiriquinus, recovered on August 15, 2013 at the Autopista del Café, El Roble, Quindío, apparently hit by a car. The specimen corresponds to the first record of the Colección Mastozoológica de la Universidad del Quindío (CMUQ), identified with catalogue number CMUQ-001. Despite the species has been already reported for the department, the herein documented specimen constitutes the first verified record through a scientific procedure in a peer-reviewed publication. Finally, we discus on the importance of the beginning of an official collection of mammals at the Universidad del Quindío.
\end{abstract}

Keywords: Eptesicus chiriquinus, Mammals Quindío, Scientific collection, Vespertillionidae.

Con el registro de un especímen del murciélago vespertilionido Eptesicus chiriquinus, se da inició a la Colección Mastozoológica de la Universidad del Quindío (CMUQ), iniciativa con la que se espera aportar a la documentación del patrimonio biodiverso de la región del Eje Cafetero en los Andes Centrales de Colombia, esperando también se convierta en fuente de referencia e investigación mastozoológica y sus disciplinas asociadas para muchas generaciones de científicos quindianos y de otras regiones del país y el exterior.
El departamento del Quindío a pesar de ser el más pequeño en área $\left(1845 \mathrm{~km}^{2}\right)$ del territorio colombiano, se ubica en una posición privilegiada en los Andes Centrales, cubriendo un amplio rango altitudinal entre los 1190 y 5200 metros de elevación (Páramo del Quindío) y su área se extiende sobre los valles de dos de los ríos más importantes del país, río Magdalena (oriente) y río Cauca (occidente). Además, su territorio es punto de encuentro de varias bioregiones y distritos biogeográficos de Colombia (HernándezCamacho et al. 1992). Como es de esperarse, la

\footnotetext{
Programa de Biología, Universidad del Quindío, Armenia, Colombia. e-mail: diegofgutierrezc@gmail.com Fecha recepción: Noviembre 4, 2013 Fecha aprobación: Diciembre 11, 2013 Editor asociado: Jiménez AM
} 
mastofauna del Quindío es diversa y su territorio incluye la localidad tipo de algunos taxones (i. e. Sciurus granatensis quindianus; J. A. Allen 1914), así como 13 especies identificadas con algún grado de amenaza en el libro rojo de mamíferos de Colombia (Rodríguez-Mahecha et al. 2006).

A pesar de la importancia de la riqueza biótica del departamento, a la fecha no se cuenta con una colección mastozoológica local que permita efectuar de una mejor y más efectiva manera, la documentación y consulta del patrimonio biodiverso del departamento en lo referente a este grupo de vertebrados. De otra parte, la ausencia de registros de mamíferos verificados en la literatura es una situación común para muchas de las especies putativamente asociadas con la fauna del departamento del Quindío, aspecto que de múltiples maneras dificulta la investigación y el desarrollo de planes de manejo y conservación de la fauna del departamento. La Universidad del Quindío ha identificado este vacío y en consecuencia se han adelantado acciones concretas para suplirlo, siendo esta institución donde, de manera histórica, por primera vez en Colombia se abre una plaza para cubrir el área específica de la mastozoología, iniciativa que surgió directamente de los estudiantes del Programa de Biología. En la Universidad opera desde hace 10 años el Grupo de Estudio en Mamíferos Silvestres (GEMSUQ), grupo de estudiantes con una de las trayectorias más amplias y continuas en la mastozoología en Colombia. La iniciativa de la Colección de Mamíferos ha sido acogida con entusiasmo por parte de la Vicerrectoría de Investigación de la Universidad. Con el inicio de la colección se articula una pieza más en la consolidación de la mastozoología regional en la ecoregión del Eje Cafetero en Colombia.

Los registros departamentales son de alta importancia en conservación desde que en Colombia, según la Ley 99 de 1993, se establece que las Corporaciones Autónomas Regionales (CAR) tienen la responsabilidad de implementar y ejecutar las políticas de carácter ambiental en el contexto regional, siguiendo directrices del Ministerio del Medio Ambiente y Desarrollo Sostenible, creado a través de la Ley 3570 de 2011. Los planes generados por las CAR se nutren entre otras fuentes, de información depositada en las colecciones científicas. En este sentido se espera que con la iniciativa de la colección se refuerce el vínculo ya existente entre la Universidad del
Quindío y la Corporación Regional del Quindío (CRQ). Esta relación entre las CAR y las colecciones científicas se identifica como fundamental e ineludible. Más allá de representar soporte científico para la planeación y tomar las decisiones de conservación a nivel local, las colecciones científicas representan un registro en el tiempo que permite el monitoreo del estado de la diversidad regional, lo que constituye un aspecto fundamental de las acciones a ser desarrolladas también por la Gobernación Departamental y sus alcaldías.

Registro museológico. En este trabajo se corrobora la presencia de E. chiriquinus para el departamento del Quindío, registro que representa no solamente una extensión geográfica y ecológica de 186 $\mathrm{km}$, con respecto al registro más cercano (en una publicación revisada por pares) sobre el sistema andino colombiano, proveniente de Pance, Valle del Cauca (Davis y Gardner 2008), verificando la hipótesis propuesta en la tesis de maestría de Ramírez-Chávez (2008), sobre la presencia de la especie en el departamento.

Presencia de Eptesicus chiriquinus para el departamento de Quindío, Colombia. El murciélago vespertilionido $E$. chiriquinus tiene una amplia distribución en el neotrópico, encontrándose desde México, a través de Centro América, Colombia, Venezuela, Guyana, Brasil, Ecuador, Perú, hasta Bolivia (Davis y Gardner 2008). Aunque para Colombia se cuenta con registros museológicos de E. chiriquinus reportados para los departamentos de Antioquia, Caldas, Caquetá, Cauca, Cundinamarca, Quindío, Risaralda, Santander, y Valle del Cauca, los únicos registros de esta especie para el Quindío, corresponden a los analizados por Ramírez-Chávez (2008) en su tesis de maestría, trabajo que se cita en Solari et al. (2013) como referencia de la especie para el departamento.

Atropellamiento de murciélagos en la Autopista del Café. Como parte del estudio «Mortalidad de fauna silvestre en la Autopista del Café»» se recuperó un macho juvenil del género Eptesicus, el 15 de agosto de 2013, en el tramo que comunica la ciudad de Armenia con la ciudad de Pereira, a la altura del municipio de Circasia a una distancia de $2,7 \mathrm{~km}$ en sentido norte-sur del restaurante El Roble (N 04³8'52.3' W 075³6'14.5”) (Figura 1); este ejemplar que fue aparentemente atropellado la noche in- 
mediatamente anterior a su recolección. El espécimen recuperado fue contrastado tanto en sus características externas como internas con las claves, caracteres y medidas propuestos en Muñoz-Arango (2001), Davis (1966), Davis y Gardner (2008), Miller (1907), Ramírez-Chávez (2008), y Tirira (2007) (Figura 1) y fue preservado como carcasa y piel en fluido, cráneo extraído, depositado en la Colección Mastozoológica de la Universidad del Quindío (CMUQ) e identificado como E. chiriquinus con número de catálogo CMUQ-001 (tejidos asociados).

Caracteres externos. El espécimen CMUQ-001 presentó un antebrazo $(\mathrm{AB}) 46.73 \mathrm{~mm}$, ubicándolo dentro de las especies grandes del género Eptesicus
( $\mathrm{AB}>42.0 \mathrm{~mm}$ ); presenta un pelaje de longitud media $8.2 \mathrm{~mm}$; dorso de color marrón oscuro (Burnt umber, lámina XXVIII 9" m, Ridgeway 1912), cabeza pronunciada, hocico hinchado, que da la apariencia de estar algo inflamado (Figura 2), orejas de tamaño medio (12 mm); trago largo y comprimido (7 $\mathrm{mm}$ ), algo redondeado en la punta, ojos pequeños, calcáneo largo $(9.14 \mathrm{~mm})$, superando la longitud del pie $(8.54 \mathrm{~mm})$.

Caracteres internos. La longitud máxima del cráneo fue de $15.66 \mathrm{~mm}$, estando ligeramente por debajo del límite sugerido por Davis y Gardner (2008) para la especie (LMC $>15.8 \mathrm{~mm}$ ); la longitud de la fila superior de dientes fue de $6.18 \mathrm{~mm}$; cráneo con

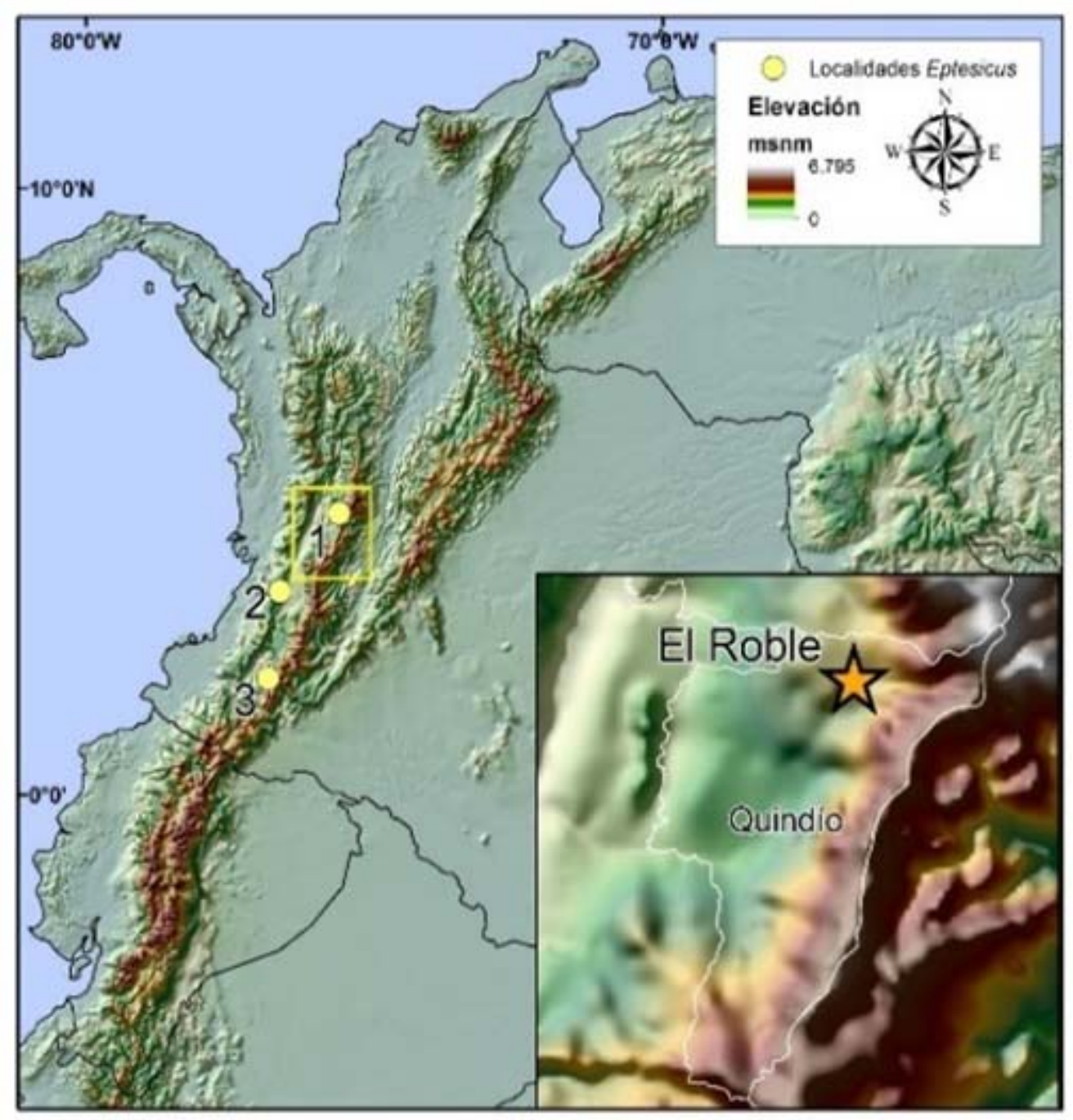

Figura 1. Mapa de localidades marginales en la distribución occidental de E. chiriquinus en Colombia: 1) El Roble, Quindío; 2) Pance, Valle del Cauca; y 3) Almaguer, Cauca. Recuadro señalando la ubicación del registro de E. chiriquinus para el departamento del Quindío (estrella amarilla). 

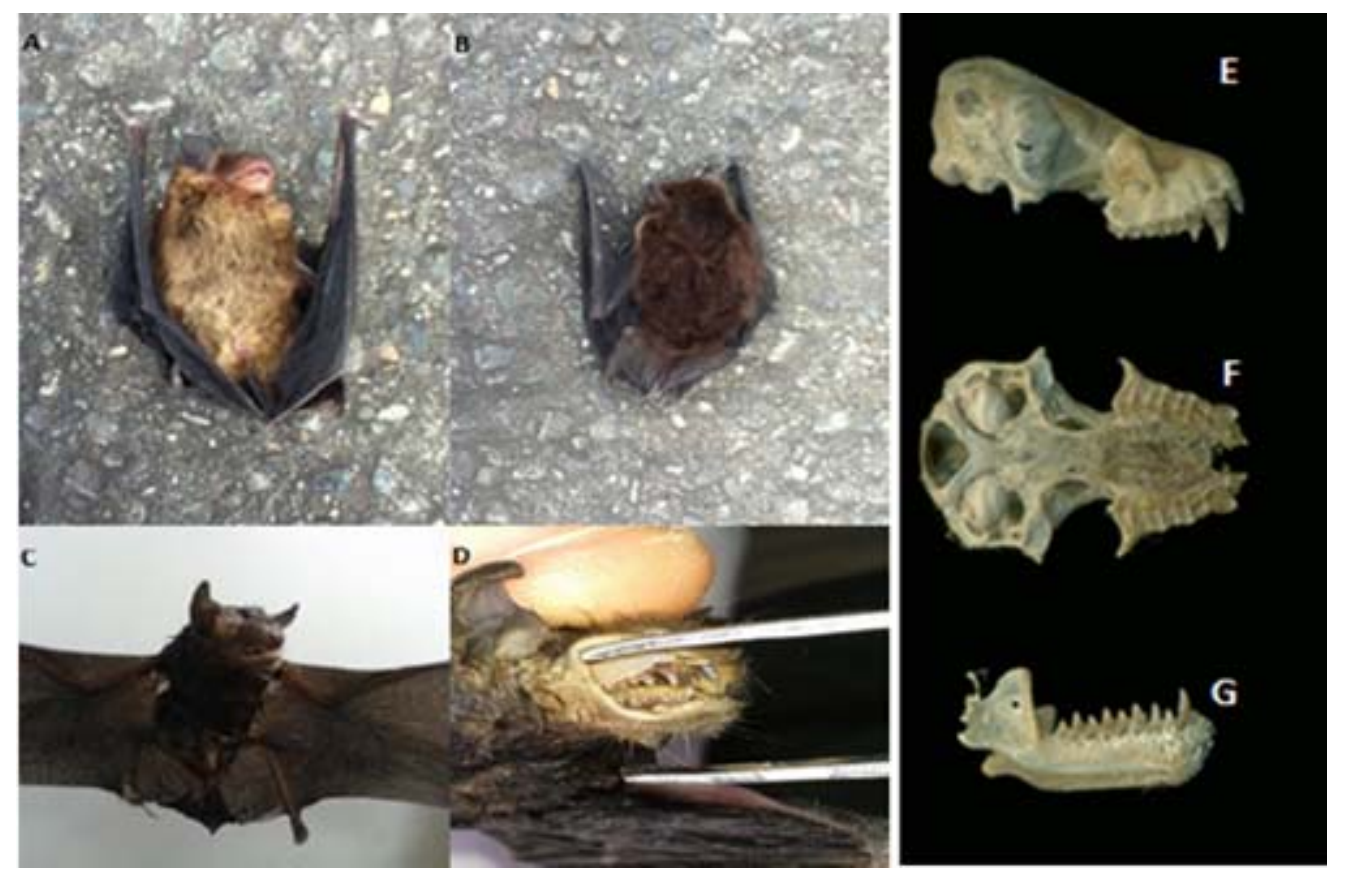

Figura 2. A y B. Vista ventral y dorsal del espécimen; C. Vista frontal en la que se observa la apariencia inflamada del hocico, característica típica del genero Eptesicus; D. Vista lateral de la fila superior de dientes, mostrando un único premolar grande que no deja un espacio libre detrás del canino; E. Vista lateral del cráneo; F. Vista ventral del cráneo; G. Vista lateral de la mandíbula.

presencia de una cresta sagital y cresta lambdoidea bien desarrolladas (Figura 2E). En vista lateral de la fila superior de dientes muestra un único premolar grande que no deja un espacio libre detrás del canino (Figura 2D). En la Tabla 1 se muestran las medidas del espécimen recuperado y se comparan con las realizadas por Ramírez-Chávez (2008) en su revisión de representantes de E. chiriquinus y E. brasiliensis para Colombia.

Consideraciones finales. Se reconoce como importante el desarrollo de trabajos de recuperación de especímenes de fauna atropellada, los cuales aportan información espaciotemporal de las especies en un área determinada. En este orden de ideas, el encuentro de este ejemplar de E. chiriquinus sobre la malla asfáltica, nos alerta sobre potenciales efectos que la generación de esta infraestructura pueda tener sobre las poblaciones naturales de quirópteros. Producto de esta investigación se han registrado al menos otros seis episodios de atropellamiento de quirópteros, los cuales no pudieron ser determinados debido al mal estado de los especímenes.

\section{Agradecimientos}

Agradecemos de manera especial a P. Landázuri, Vicerrectoría de Investigación de la Universidad del Quindío y a C. A. Agudelo-Henao, Dirección del Programa de Biología de la Universidad del Quindío por el apoyo en la iniciativa de la Colección de Mamíferos de la Universidad del Quindío. A H. RamírezChávez por su asesoría en la verificación taxonómica; al Grupo de Estudio en Mamíferos Silvestres de la Universidad del Quindío (GEMSUQ); a A. Botero por su asesoría en el desarrollo del Proyecto «Fauna atropellada en la Autopista del Café».

\section{Literatura citada}

Davis WB. 1966. Review of South American bats of the genus Eptesicus. Southwest Nat. 11 (2): 245-74.

Davis WB, Gardner AL. 2008. Genus Eptesicus Rafinesque, 1820. In: Gardner AL (ed.). Mammals of South America. Volume 1. Marsupials, xenarthrans, shrew, and bats. Chicago: The University of Chicago Press; 669 pp.

Gardner AL. 2008. [2007]. Mammals of South America. Volume I: Marsupials, xenarthrans, shrews, and bats. Chicago: 
Tabla 1. Comparación de las medidas del espécimen de Eptesicus chiriquinus CMUQ 001 con las reportadas por Ramírez-Chávez (2008) para E. chiriquinus y E. brasiliensis. Medidas en milímetros.

\begin{tabular}{|c|c|c|c|}
\hline \multirow[t]{2}{*}{ Eptesicus chiriquinus } & \multicolumn{2}{|c|}{ E. chiriquinus } & \multirow{2}{*}{$\begin{array}{c}\text { E. brasiliensis } \\
\text { Ramírez-Chávez (2008) }\end{array}$} \\
\hline & CMUQ-001 & Ramírez-Chávez (2008) & \\
\hline Longitud mayor del cráneo & 15.96 & $17,27(16,70-18,12)(N=7)$ & $16,48(15,70-17,12)(\mathrm{N}=26)$ \\
\hline Longitud máxima del cráneo sin incisivos & 15.66 & $16,59(15,82-17,17)(N=7)$ & $15,83(15,04-16,85)(N=27)$ \\
\hline Longitud cóndilo-canino & 14.45 & $15,65(15,18-16,09)(N=7)$ & $14,62(14,18-15,01)(N=26)$ \\
\hline Longitud cóndilo-incisivo & 15.72 & $16,22(15,43-17,05)(N=7)$ & $15,30(14,74-16,18)(N=26)$ \\
\hline Longitud basicraneal & 13.16 & $13,93(13,38-14,4)(N=6)$ & $12,84(12,41-13,30)(N=25)$ \\
\hline Longitud del paladar & 6.97 & $7,39(7,25-7,73)(N=7)$ & $6,92(6,38-7,75)(\mathrm{N}=26)$ \\
\hline Ancho del rostro & 4.37 & $5,51(5,08-5,83)(\mathrm{N}=8)$ & $5,36(4,86-5,95)(\mathrm{N}=28)$ \\
\hline Constricción postorbital & 4.09 & $4,13(3,96-4,38)(\mathrm{N}=8)$ & $3,81(3,45-4,51)(N=28)$ \\
\hline Alto de la bóveda craneana & 6.13 & $6,21(5,67-6,75)(\mathrm{N}=7)$ & $5,85(5,32-6,49)(\mathrm{N}=27)$ \\
\hline Ancho de la bóveda craneana & 7.45 & $7,70(7,40-8,19)(\mathrm{N}=8)$ & $7,34(6,92-7,92)(N=27)$ \\
\hline Ancho mastoideo & 8.64 & $8,70(8,44-8,97)(\mathrm{N}=7)$ & $8,34(7,67-8,69)(\mathrm{N}=27)$ \\
\hline Ancho zigomático & 10.03 & $11,27(10,67-11,64)(\mathrm{N}=6)$ & $10,74(9,99-11,34)(N=17)$ \\
\hline Constricción anterorbital & 5.49 & $5,57(5,13-5,82)(\mathrm{N}=8)$ & $5,36(4,75-5,85)(N=28)$ \\
\hline Ancho entre los caninos & 4.98 & $5,06(4,51-5,4)(N=8)$ & $4,85(4,05-5,23)(N=27)$ \\
\hline Mayor ancho entre los molares superiores & 7.03 & $7,15(6,96-7,36)(\mathrm{N}=8)$ & $6,70(5,81-7,22)(\mathrm{N}=28)$ \\
\hline Longitud de la serie dental superior con canino & 6.18 & $6,41(6,12-6,63)(\mathrm{N}=8)$ & $5,87(5,09-6,15)(N=27)$ \\
\hline Longitud de la serie dental superior con incisivos & 7.12 & $7,42(7,11-7,83)(\mathrm{N}=8)$ & $6,82(5,79-7,18)(\mathrm{N}=25)$ \\
\hline Longitud de la serie dental inferior con caninos & 7.01 & $6,98(6,53-7,37)(\mathrm{N}=8)$ & $6,46(5,89-7,17)(\mathrm{N}=26)$ \\
\hline Longitud de los molariformes superiores & 4.94 & $5,02(4,77-5,22)(\mathrm{N}=8)$ & $4,70(4,44-4,88)(\mathrm{N}=26)$ \\
\hline Longitud de los molariformes inferiores & 5.27 & $5,98(5,53-6,38)(N=8)$ & $5,63(5,35-6,53)(\mathrm{N}=26)$ \\
\hline Longitud de la mandíbula & 12.43 & $13,17(12,85-13,61)(N=7)$ & $12,33(11,46-13,67)(\mathrm{N}=26)$ \\
\hline Altura de la mandíbula & 4.85 & $5,10(4,57-5,44)(\mathrm{N}=7)$ & $4,99(4,42-5,49)(N=25)$ \\
\hline Longitud oreja & 12 & $12,13(9,00-16,00)(\mathrm{N}=8)$ & $13,00(9,00-19,00)(N=29)$ \\
\hline Longitud trago & 7 & $6,67(5,93-7,50)(N=7)$ & $6,53(5,03-7,93)(\mathrm{N}=30)$ \\
\hline Longitud del antebrazo & 46.73 & $44,67(42,45-47,62)(N=8)$ & $40,41(37,56-44,85)(N=33)$ \\
\hline Longitud del tercer metacarpal & 43.51 & $41,92(39,02-45,51)(N=7)$ & $37,69(33,77-42,49)(N=31)$ \\
\hline Longitud del cuarto metacarpal & 41.15 & $39,46(36,85-42,34)(N=7)$ & $36,53(32,95-39,56)(N=31)$ \\
\hline Longitud del quinto metacarpal & 39.02 & $37,27(34,61-39,50)(\mathrm{N}=7)$ & $35,08(32,47-37,06)(N=31)$ \\
\hline Longitud del pie & 9 & $9,69(8,55-10,46)(\mathrm{N}=8)$ & $8,75(7,22-9,69)(\mathrm{N}=33)$ \\
\hline Pelaje dorsal & 8.2 & $9,29(8,00-11,00)(N=7)$ & $6,81(5,00-9,00)(N=32)$ \\
\hline Peso & 10.30 & $7,57(7,00-9,00)(\mathrm{N}=7)$ & $8,37(6,10-10,40)(N=21)$ \\
\hline
\end{tabular}

University of Chicago Press, $\mathrm{xx}+690 \mathrm{pp}$.

Hernández-Camacho JI, Hurtado A, Ortiz R, Walschburger T. 1992. Unidades biogeográficas de Colombia. En: La diversidad biológica de Iberoamérica. Halffter IG, (ed.). México: Acta Zoológica Mexicana, Instituto de Ecología, pp. 105-51.

Miller GSJr. 1907. The families and genera of bats. Smithsonian Institution, United States National Museum.
57: $195-210$.

Muñoz-Arango J. 2001. Los murciélagos de Colombia: sistemática, distribución, descripción, historia natural y ecología. Medellín: Universidad de Antioquia; 391 pp.

Ramírez-Chávez HE. 2008. Revisión taxonómica de los murciélagos del género Eptesicus Rafinesque, 1820 (Mammalia; Chiroptera: Vespertilionidae) para Colombia. (Ms: Tesis). Bogotá: Universidad Nacional 
de Colombia. 78 pp.

Ridgeway R. 1912. Color standard and color nomenclature. Washington, DC. 44 pp.

Rodríguez-Mahecha JV, Alberico M, Trujillo F, Jorgenson J. 2006. Libro rojo de mamíferos de Colombia. Serie Libros Rojos de Especies Amenazadas de Colombia. Bogotá: Conservación Internacional Colombia, Ministerio de
Ambiente, Vivienda y Desarrollo Territorial.

Solari S, Muñoz-Saba Y, Rodríguez-Mahecha JV, Defler TR, Ramírez-Chávez HE, Trujillo F. 2013. Riqueza, endemismo y conservación de los mamíferos de Colombia. Mastozool Neotrop. 20 (2): 301-65.

Tirira D. 2007. Guía de campo de los mamíferos del Ecuador. Quito: Ediciones Murciélago Blanco; 576 pp. 